\title{
Expressing Access Control Policies with an Event-Based Approach
}

\author{
Pierre Konopacki ${ }^{1,2}$, Marc Frappier ${ }^{1}$, and Régine Laleau ${ }^{2}$ \\ 1 GRIL, Département d'informatique, Université de Sherbrooke, Canada \\ 2 Université Paris-Est, LACL, IUT Sénart Fontainebleau, Fontainebleau, France \\ \{pierre.konopacki, marc. frappier\}@usherbrooke.ca, laleau@u-pec.fr
}

\begin{abstract}
Information systems are widely used and help in the management of huge quantities of data. Generally, these data are valuable or sensitive, their access must be restricted to granted users. Security is a mandatory requirement for information systems. Several methods already exist to express access control policies, but few of them support all kinds of constraints that can be defined in access control policies. In this paper, we present $\mathrm{EB}^{3} \mathrm{SEC}$, a language used to formally model and interpret access control policies in information systems. Permissions, prohibitions and static separation of duty are specified by a class diagram. As $\mathrm{EB}^{3} \mathrm{SEC}$ includes a process algebra, dynamic access control constraints such as obligations and dynamic separation of duty can be easily expressed. Finally, we present the architecture of the tool used to interpret $\mathrm{EB}^{3} \mathrm{SEC}$ models.
\end{abstract}

Keywords: formal method, access control, security.

\section{Introduction}

Information Systems (IS) are widely used in various economical and social areas. They contain private and valuable data for their owners. In IS, security is enforced by different mechanisms, such as secured protocols between clients and servers or between servers in a distributed architecture. One common purpose of these tools is to enforce an access control (AC) policy. An AC policy is part of a security policy that deals with authorizations granted to users. An AC policy badly defined can lead to major issues for the company that uses the IS: for example we can cite banks that lost billions since a trader used more authorizations that he should have been granted [1]. After these scandals, governments of different nations decided to create new laws to protect information by regulating their access. In the financial domain, we can cite the Mer law in France [2] and Sarbanes-Oxley law in USA [3]. To comply with these laws, companies have to precisely define their AC policies.

Generally, AC constraints are classified into four categories: i) Permissions allowing the execution of an action, ii) Prohibitions forbidding the execution of an action, iii) Obligations linking two actions: if a person performed one of them, he must execute the second action [4], iv) Separation of duty (SoD) linking two actions: once a person performed one of them, he is not allowed to perform the second action.

We can also differentiate them according to their static or dynamic feature. Static constraints can be considered as invariants on the system state. They are always true

C. Salinesi and O. Pastor (Eds.): CAiSE 2011 Workshops, LNBIP 83, pp. 607621, 2011.

(c) Springer-Verlag Berlin Heidelberg 2011 
whatever the state. Permissions, prohibitions and static SoD are static constraints. A static SoD constraint means that if a user is assigned to one role, he is prohibited from being a member of a second role [5]. Dynamic constraints require to take into account the history of the system, that is the set of actions already performed on a system. Obligations and dynamic SoD are dynamic constraints. With dynamic SoD, users may be authorized for roles that may conflict, but limitations are imposed while the user is actively logged onto the system.

In this article we propose a new formal specification language, called $\mathrm{EB}^{3} \mathrm{SEC}$, which aims to model AC policies. The main objectives of the language are: i) to enable the definition of the four categories of AC constraints, ii) to be supported by tools such as formal verifiers or interpreters. $\mathrm{EB}^{3} \mathrm{SEC}$ is based on the $\mathrm{EB}^{3}$ language, a process algebra language. This kind of languages is more suitable to express event ordering constraints, compared to state-based languages like B or Z [6]. The paper describes first how the four kinds of $\mathrm{AC}$ constraints can be expressed in $\mathrm{EB}^{3} \mathrm{SEC}$ and then presents the associated interpreter which is an extension of the $\mathrm{EB}^{3}$ interpreter. $\mathrm{EB}^{3} \mathrm{SEC}$ is currently used in projects led with companies from financial domain 1 and healthcare domain 2 .

Section 2 presents the $\mathrm{EB}^{3}$ method and a case study, inspired from a real case study provided by our industrial partner; it has been simplified for illustrative purposes. Section 3 presents how we can model AC policies with the $\mathrm{EB}^{3}$ SEC method. Section 4 describes the $\mathrm{EB}^{3} \mathrm{SEC}$ interpreter. Section 5 compares $\mathrm{EB}^{3} \mathrm{SEC}$ to other similar languages. Finally, we conclude in Section 6, outlining future work.

\section{Background}

\subsection{An Overview of $\mathrm{EB}^{3}$}

$\mathrm{EB}^{3}$ [7] (entity-based black box) is a formal language for the specification of IS. An $\mathrm{EB}^{3}$ specification contains four parts. A requirements class diagram describes classes and associations of the IS. A process expression (PE), denoted by main(, ) describes valid input traces that must be accepted by the IS. A trace is a sequence of events. An event corresponds to an instanciation of an action of the IS. The other parts are omitted in this paper since they are not needed to understand the presentation of $\mathrm{EB}^{3} \mathrm{SEC}$. The $\mathrm{EB}^{3}$ process algebra is similar to several other process algebras like CSP, CCS, ACP and Lotos. We shall briefly introduce it here; the reader is referred to [7] for a complete description.

Complex $\mathrm{EB}^{3}$ process expressions (PE) are constructed from elementary process expressions (actions) using the following operators:

- The Kleene closure written ${ }^{*}$ is used to iterate a process expression an arbitrary number of time.

- The choice which is denoted by | and expresses that one of two process expressions can be executed.

\footnotetext{
${ }^{1} \mathrm{http}: / /$ pages.usherbrooke.ca/eb3sec/

$2 \mathrm{http}: / /$ lacl.univ-paris $12 . \mathrm{fr} /$ selkis/
} 
- The quantified choice is a variant of the previous operator. When a process expression depends on the value of a free variable, a quantified choice can be used to express the fact we can execute this process expression for a specific value of the free variable. It is denoted by: $\mid x \in$ ens $: \mathbf{a}(x)$, and it means that $\mathbf{a}(x)$ is executed with a specific value of $x$ taken in the set ens.

- The wildcard _ is a syntax shortcut used instead of a quantified choice. For example, $a(-)$ means $\mid x \in$ ens : $\mathbf{a}(x)$

- The guard denoted by $p \Longrightarrow a$ where $p$ is a first order logic predicate and $a$ is a process expression, allows one to execute $a$ only if the predicate $p$ holds. If $p$ does not hold, the process expression waits (blocking behavior) until $p$ becomes true.

- The sequence denoted by $\mathbf{a} \cdot \mathbf{b}$ allows one to execute the process expression $\mathbf{b}$ after the execution of the process expression $\mathbf{a}$.

- The parallel denoted by $\mathbf{a} \| \mathbf{b}$ allows one to execute the process expression $\mathbf{a}$ and the expression $\mathbf{b}$ in parallel with synchronization on common actions.

- The interleaving denoted by $\mathbf{a} \| \mathbf{b}$ allows process expressions $\mathbf{a}$ and $\mathbf{b}$ to be executed in parallel without any synchronization, even if they have common actions.

- The quantified interleaving is denoted by $\| \mid p \in$ ens $: \exp (p)$. It corresponds to the interleaving of process expressions $\exp (p)$ for all values of $p$ contained in the set ens.

The main differences between $\mathrm{EB}^{3}$ and CSP are: i) $\mathrm{EB}^{3}$ allows one to use a single state variable, the system trace, in predicates of guard statements (as we shall see below); ii) $\mathrm{EB}^{3}$ uses a single operator, concatenation (as in regular expressions), instead of prefixing and sequential composition, which makes specifications easier to read and write.

$\mathrm{EB}^{3}$ is implemented in a platform called APIS that allows its interpretation. Section 4 will described more precisely this platform.

\subsection{The Running Example}

The example used to illustrate the article deals with a check deposit process in a bank. A bank client wants to deposit a check on his account. To start the check deposit procedure, the client has to bring the check to his bank branch where a bank employee starts the procedure. The check is first entered in the system and then registered to the client. The check is then validated or cancelled. If the check is validated the account of the client is credited, otherwise the client's account is not credited.

To prevent frauds, actions of a check deposit procedure must be delegated to different persons. We provide below a typical natural description of security rules that can be found in a bank.

- rule 1: Only clerks and bankers are allowed to make check deposit effective.

- rule 2: Only bankers and directors of branch are allowed to cancel or validate a check deposit.

- rule 3: Only clerks and bankers are allowed to modify the amount of a bank account.

- rule 4: The validation or the cancellation of a deposit cannot be done by the same person that did the check deposit. 
- rule 5: If the value of a check deposit exceeds a given amount, the check deposit must be validated by two different persons including the director of branch. In Quebec this amount is equal to $10000 \$$ whereas in Ontario this amount is equal to 8000 \$.

- rule 6: The modification of the bank account of the client must be done by the same employee that did the check deposit effective, with the same role (clerk or banker) in the same branch.

In this example, four different actions are used: deposit, cancel, validate credit. These actions have three arguments, which refer to the client, the identifier of the check and the value of the check deposit. In this example, a person can play different roles: clerk, banker and branch-director. We also assume that we have at least two bank branches one in Montreal, QC and the other in Toronto, ON.

\section{Modeling Access Control Policies}

\section{1 $\mathrm{EB}^{3} \mathrm{SEC}$}

The $\mathrm{EB}^{3}$ SEC language uses the $\mathrm{EB}^{3}$ operators to specify valid input traces of secure events. A secure event is composed of an $\mathrm{EB}^{3}$ event enhanced with security parameters representing persons, roles, organizations... The originality of $\mathrm{EB}^{3} \mathrm{SEC}$ versus other $\mathrm{AC}$ languages is that the number and the type of security parameters can be adapted to the IS. Thus $\mathrm{EB}^{3} \mathrm{SEC}$ allows the designer to define his own AC model. It can be existing models such as Bell and LaPadula [8], RBAC [9] or OrBAC [10]... or extensions of them in order to capture specific AC requirements of the IS. The AC model defined for the running example is derived from the RBAC model. Moreover, as we need to represent the different branches of a bank, we also used the concept of organization presented in OrBAC. We could represent this concept in RBAC thanks to the role concept. However it would duplicate the number of roles because, in our case, roles are the same in any branches. Security parameters chosen for the running example are: person, role, branch and time. Thus, a secure event is represented by a 5-tuple:

$\langle p, r, o, t, e v t\rangle$ where $p \in$ Person, $r \in$ Role, $o \in$ Branch, $t \in$ Timestamp, label $($ evt $) \in$ Action

Function label returns the label of an event (e.g. label(deposit(...)) = deposit).

In $\mathrm{EB}^{3} \mathrm{SEC}$, the following kinds of $\mathrm{AC}$ constraints can be expressed:

- Permission allows the execution of an action.

- Prohibition forbids the execution of an action.

- Obligation links two actions. Once a person performed one of them, he must execute the second action.

- Separation of duty (static and dynamic) links two actions. Once a person performed one of them, he is not allowed to perform the second action. 
The class diagram of an $\mathrm{EB}^{3} \mathrm{SEC}$ specification is used to express static $\mathrm{AC}$ constraints. Process expressions are used to express dynamic AC constraints. Thus, static and dynamic AC constraints can be expressed in the same language. Furthermore, another advantage is that consistency of $\mathrm{EB}^{3} \mathrm{SEC}$ models can be verified with model checking tools.

\subsection{Permission and Prohibition}

In comparison with other $\mathrm{AC}$ modeling languages, $\mathrm{EB}^{3} \mathrm{SEC}$ allows permissions and prohibitions to be expressed with the same level of expressiveness as [11[10[12].

The class diagram presented in figure 1 describes permissions and prohibitions of the running example. The class Person lists the different persons of the IS. The class Role represents the roles that can be played in the IS. In the example, they are: clerk, banker, branch-director. The class Branch encompasses all branches of the IS (i.e., Montreal, Toronto). The association Play linking these three classes depicts roles played by persons in the different branches. The class Action contains all actions that can be executed in the IS. The associations Permission and Prohibition associate a subset of actions of the class Action to a role in a branch. For Permission (resp. Prohibition) it gives the actions that a role is allowed (resp. not allowed) to execute.

Note that, even if it is not considered in the running example, $\mathrm{EB}^{3} \mathrm{SEC}$ allows inheritance of roles or hierarchies of organizations to be defined in the security class diagram.

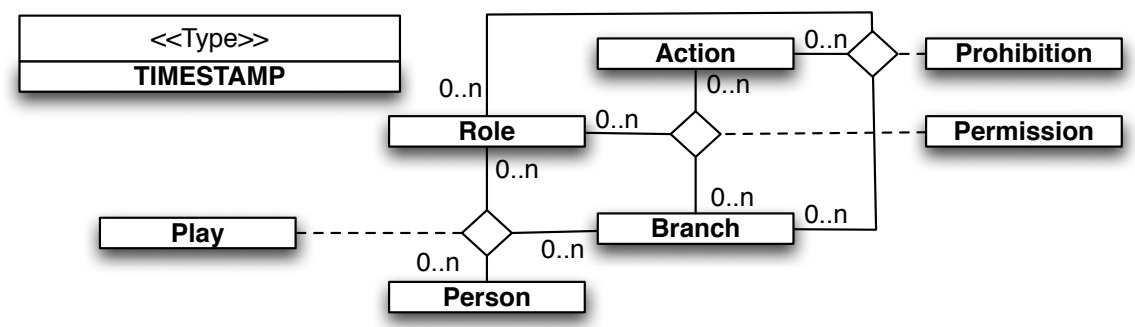

Fig. 1. Security class diagram for the running example

In our case study, the rules Rule 1, Rule 2 and Rule 3 concern permissions. Figure 2 gives an instanciation of the association Permission for Rule $\mathbf{1}$.

The security class diagram must be supplemented with a predicate that determines how an event (i.e., an action executed by a user) satisfies the permissions and prohibitions described in the security class diagram. The static predicate $s p(\langle p, r, o, t, e v t\rangle)$ is built using the parameters of the security event and the classes and associations of the security class diagram. For the bank example, this predicate is the following:

$$
\begin{aligned}
s p(\langle p, r, o, t, \text { evt }\rangle) \triangleq & \langle p, r, o\rangle \in \text { play } \wedge \\
& \langle r, \text {, label }(\text { evt })\rangle \in \text { permission } \wedge \\
& \langle r, o, \text { label }(\text { evt })\rangle \notin \text { prohibition } \wedge \\
& t \in \text { TIMESTAMP }
\end{aligned}
$$




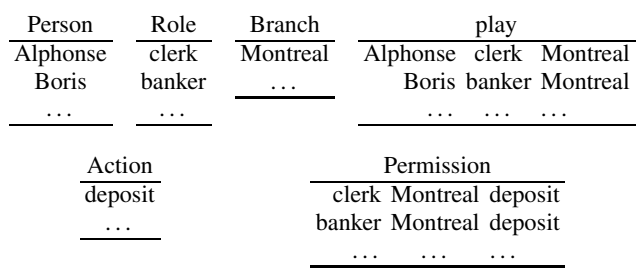

Fig. 2. Instantiation of the security class diagram for the running example

This predicate states that a security event of the form $\langle p, r, o, t, e v t\rangle$ (from the signature above) holds iff the triple $\langle p, r, o\rangle$ is an instance of association Play, the triple $\langle r, o$, label (evt $)\rangle$ is an instance of association Permission and the triple $\langle r, o$, label $($ evt $)\rangle$ is not an instance of association Prohibition.

We omit here the $\mathrm{EB}^{3} \mathrm{SEC}$ syntax for defining predicate $s p$. It is similar to the syntax used for predicates in attribute definitions and guards in $\mathrm{EB}^{3}$. Essentially, the predicate $s p$ can refer to any class and association attributes. Usual logical connectors and quantifiers are supported: $\wedge, \vee, \neg, \Rightarrow, \Leftrightarrow, \forall, \exists$. Transitive closure on recursive associations is also supported, in order to deal with inheritance and hierarchical data (e.g. hierarchical roles, hierarchical group of actions, hierarchical organizations).

$\mathrm{EB}^{3} \mathrm{SEC}$ is an $\mathrm{AC}$ modeling language that allows one to specify both permissions and prohibitions. This feature is very useful but can be very dangerous too. Indeed, it can lead to permissions and prohibitions that contradict each other. Model checking tools can be used to verify the consistency of the model, but it is strongly advised to use permissions or prohibitions only.

Permissions and prohibitions used in the running example do not contain any constraints. EB ${ }^{3}$ SEC can also be used to model AC policy containing permissions and prohibitions with constraints. Examples of such AC rules can be found in [13].

\subsection{Obligation}

Contrary to permissions and prohibitions, the concept of obligation is not precisely defined in the literature. Roughly speaking, each AC language gives its own definition. In XACML [12], an obligation is always associated to a permission. If the obligation cannot be fulfilled, the permission is denied. In [10[9]4], it refers to an organizational constraint that obliges the user to perform another action after, before or while executing another one. For instance, we can express an obligation for a person to execute an action of acknowledgement after performing an action. Typically, it is the kind of constraints expressed in the sixth rule.

Basically, an obligation generally involves an ordering constraint between a number of security events, linked by the value of at least one security parameter (i.e., the person ...). Thus it can be expressed in $\mathrm{EB}^{3} \mathrm{SEC}$ by a process expression.

For example, Rule 6 is specified by:

\section{1. $\operatorname{Rule6}() \triangleq$}

2. $\| \mid c \in$ Customer : $\| d \in$ int : $\| m \in$ int : 
3.

$\mid p \in$ Person $: \mid r \in$ Role $: \mid o \in$ Branch :

4.

$\left(\left\langle p, r, o_{-}, \operatorname{deposit}(c, d, m)\right\rangle\right.$

5.

- $\left.\left\langle p, r, o,,_{-}, \operatorname{credit}(c, d, m)\right\rangle\right)^{*}$

The second line contains the quantifications over functional parameters, in other words, it can be translated by: For each customer c, for each check deposit with the number $d$ and the amount $m$. The third line contains quantifications related to the security class diagram and can be translated by: there exist a person $p$, a role $r$ and a branch $o$. 5-tuples of the fourth and fifth lines stipulate that this person $p$ can play the role $r$ in the branch $o$ and that, for this role and branch, actions deposit and credit are permitted and not prohibited. Furthermore, the sequence expresses that the deposit and the credit must be done by the same person playing the same role in the same branch. Suppose that the security event $\langle$ adrian, clerk, Montreal, deposit $(z o e, 1,23)\rangle$ has already been executed. Thus, a security event corresponding to the event $\operatorname{credit}(z o e, 1,23)$ can be performed only by adrian playing the role clerk in the branch of Montreal.

The process expression only expresses that the two actions have to be done by the same person with the same role in the same branch. However the system cannot physically force the person to perform the second action after executing the first one. We propose two methods that can be acceptable solutions for this problem.

A first method to enforce the obligation is to forbid any action, other than the second action, for the person after he executed the first one. It is modeled by the following PE.

\section{Rule6bis ()$\triangleq$}

2. $\| \mid c \in$ Customer : $\| d \in$ int : $\| m \in$ int :

3. $\mid p \in$ Person: $\mid r \in$ Role $: \mid o \in$ Branch :

4. $\quad\left(\left\langle p, r, o_{-}, \operatorname{deposit}(c, d, m)\right\rangle\right.$

5. $\quad$. $\quad(\langle p, r, o,-, \operatorname{credit}(c, d, m)\rangle$

6.

7.

8.

FALSE $\Longrightarrow$

$$
\left\langle p, r, o_{-,},{ }_{-} \text {deposit }\left(_{-,-},-\right)\right\rangle
$$

9.

10.

$$
\text { | FALSE } \Longrightarrow
$$

11.

$$
\begin{aligned}
& \left\langle p, r, o_{-},-, \text {cancel }\left({ }_{-},-,-\right)\right\rangle \\
& \text {|FALSE } \Longrightarrow \\
& \left.\left.\left\langle p, r, o_{,-,}, \text {validate }\left({ }_{-},{ }_{-},-\right)\right\rangle\right)\right)^{*}
\end{aligned}
$$

In this PE, after performing the action deposit, the person has the choice between four actions. Three of these four actions are blocked by a guard that will never hold. Thus, the person can perform only the action deposit for the check deposit he performed.

The second approach used in [10], consists in adding sanctions to enforce obligations. In other words, the second action of the obligation is guarded by a constraint (generally temporal). While the guard holds, the person can execute the second action, but once it does not hold any more the sanction is executed. In Rulebter, a second version of Rule 6 called Rule 6 ter is given. Rule 6 ter adds to the sixth rule a temporal constraint: the modification has to be executed at least one hour after the deposit.

\section{1. $\operatorname{Rule6ter}() \triangleq$}

2. $\| \mid c \in$ Customer : $\| \mid d \in$ int : $\| m \in$ int : 


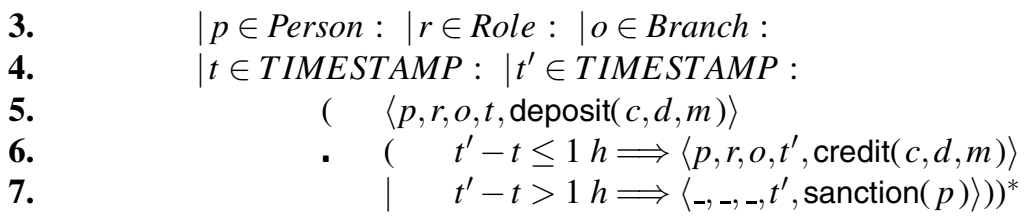

Once the person performed the action deposit, there is a choice between two guarded actions. The two guards partition the state space. Only during the hour following the deposit, the user can execute credit. After that, the only action that can be performed is $\operatorname{sanction}(p)$.

\subsection{Separation of Duty}

SoD (Separation of duty) is a security requirement that divides a task into subtasks and dispatches the execution of these subtasks to different persons [5]. Thus, the security of the process has fewer chances to be corrupted by a single person who could execute all the subtasks by himself. To express this kind of constraints, two methods can be used.

\section{Static separation of duty}

A first way to address the problem of SoD is called static. This method is used in [14 15] and consists in avoiding a person to play two different roles that are involved in the same SoD. To adapt this method to $\mathrm{EB}^{3} \mathrm{SEC}$, one has to perform a static analysis on the instantiated class diagram and check that the association Play respects this property.

In the running example, we want to express that Person alphonse cannot play the role branch-director both in branches Montreal and Toronto. This means that the two triples $\langle$ alphonse, branch - director,Montreal $\rangle$ and $\langle$ alphonse, branch-director, Toronto $\rangle$ cannot be present together in the association Play.

\section{Dynamic separation of duty}

For two actions, dynamic SoD forbids the user to execute the second action, once the first action has been executed. To achieve dynamic SoD, a history of tasks already performed needs to be registered. Methods [16[17] use this solution to implement dynamic SoD.

The fourth and fifth rules deal with dynamic SoD constraints. Rule 4 can be expressed by the following PE.

\section{Rule 4( ) $\triangleq$}

2. $\| \mid c \in$ Customer : $\| d \in$ int : $\| m \in$ int :

3. $\mid p \in$ Person : $\mid p^{\prime} \in$ Person :

4. $\quad\left(\left\langle p,_{-},,_{-}, \operatorname{deposit}(c, d, m)\right\rangle\right.$

5. $\quad \cdot p \neq p^{\prime} \Longrightarrow$

6. $\quad\left(\left\langle p^{\prime},,_{-,},-\right.\right.$, validate $\left.\left.\left.(c, d, m)\right\rangle \mid\left\langle p^{\prime},,_{-,},-, \operatorname{cancel}(c, d, m)\right\rangle\right)\right)^{*}$

The second line is a quantification over functional attributes, and means that the process expression holds for all check deposits. The third line is a quantification over security attributes that stipulates that we can find two persons. One of them has to perform the action deposit (line 4), whereas the other has to perform either the action 
cancel or the action validate (line 6). The guard (line 5) specifies that the two persons are different.

In $\mathrm{EB}^{3} \mathrm{SEC}$, dynamic SoD constraints are defined at a workflow process level. For example, a person making the deposit of a check can still do other deposit but won't be able to make the validate or the cancel for the check he deposited. We illustrate this by the following sequences of events that can be received. The two first sequences can be accepted by PE Rule 4:

$\langle$ adrian, clerk, Montreal, 1234, deposit (zoe, 1, 123)〉.

$\langle$ jenny, clerk, Montreal,4235, validate $(z o e, 1,123)\rangle$

and

$\langle$ adrian, clerk, Montreal, 1234, deposit (zoe, 1, 123)〉.

$\langle$ jenny, clerk, Montreal, 4235, deposit( arthur, 7, 51)〉.

$\langle$ adrian, clerk, Montreal, 8410, validate (arthur, 7, 51) $\rangle$.

$\langle$ jenny,clerk,Montreal,4235, cancel $(z o e, 1,123)\rangle$

whereas the third one is not admissible by PE Rule 4:

$\langle$ adrian, clerk, Montreal, 1234, deposit(zoe, 1, 123)〉.

$\langle$ adrian,clerk,Montreal, 1234, validate(zoe, 1, 123)〉

Rule 5 can be expressed by the following PE.

1. Rule 5 ( ) $\triangleq$

2. $\| \mid c \in$ Customer : $\| d \in$ int : $\| m \in$ int :

3. $\mid p \in$ Person : $\mid o \in$ Branch :

4. $\mid p^{\prime} \in$ Person: $\mid p^{\prime \prime} \in$ Person:

5. $\quad\left(\left\langle p,,_{-}, o,,_{-}, \operatorname{deposit}(c, d, m)\right\rangle\right.$.

6. $\quad((m>$ Branch.limit $(o) \Longrightarrow$

7. $\quad p \neq p^{\prime} \wedge p \neq p^{\prime \prime} \wedge p^{\prime} \neq p^{\prime \prime} \Longrightarrow$

8. $\quad\left(\left\langle p^{\prime},{ }_{-}, o,,_{-}\right.\right.$, validate $\left.(c, d, m)\right\rangle$

9. $\quad \|\left\langle p^{\prime \prime}\right.$, branch - director,,,$_{-}$, validate $\left.\left.\left.(c, d, m)\right\rangle\right)\right)$

10. $\mid(m \leq$ Branch.limit $(o) \Longrightarrow$

11. $\quad p \neq p^{\prime} \Longrightarrow\left\langle p^{\prime},{ }_{-,}, o\right.$, , validate $\left.\left.\left.(c, d, m)\right\rangle\right)\right)$

12. $)^{*}$

For checks with a value greater than the branch limit, two validate must be executed by two different persons. Otherwise there is only one validate to execute. In the example, the constraint on the check value is modeled in the PE by the expression $m>$ Branch.limit $(o)$ used in a guard, limit being an attribute of the class Branch.

\subsection{Combining AC Rules}

$\mathrm{AC}$ rules are expressed in different PE involving common actions. It remains to state, in the following PE main, how these rules are combined. 


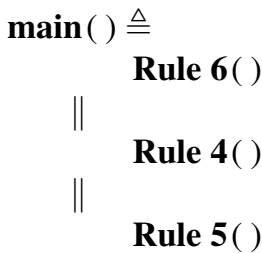

To enforce the AC policy, we must be sure that all rules are satisfied. We use the parallel composition operator $\|$ to represent the conjunction of all the ordering constraints specified in the rules. For an event to be accepted, it must satisfy each rule where it is mentioned. This is exactly what the parallel operator expresses: its operands must synchronize on common events.

Only rules corresponding to obligations and SoD are involved in the PE main. Permissions and prohibitions are enforced by the static predicate, which is checked for each secure event received.

\subsection{Discussion about $\mathrm{EB}^{3} \mathrm{SEC}$}

Different approaches can be considered to specify AC rules in $\mathrm{EB}^{3} \mathrm{SEC}$. In this paper we advocate to distinguish rules according to the type of constraints which they refer to: permissions, prohibitions, obligations or separation of duty. However it could be difficult to classify rules given by users into one of these categories since a rule can involve more than one category. Another approach consists in defining one process expression for each user rule. This will promote traceability between user requirements and the $\mathrm{EB}^{3} \mathrm{SEC}$ model.

Once an $\mathrm{EB}^{3} \mathrm{SEC}$ model is defined, some verifications can be carried out. For instance, liveness or correctness of models can be checked.

A first step of these verifications is to check that all instances of the security class diagram are useful. In our running example, the designers can check that all instances of Role could at least be activated to execute an action. This usability of all instances of Role are generalizable to all classes and associations of the diagram. With these verifications, the designer can be sure that all permissions described in the diagram could finally be used. On the opposite, he could check that all prohibitions described in the diagram are really enforced. These steps of verification ensure the correctness of the model. Furthermore, the designer can also check liveness properties over the model. Once the model is validated, it can be used as a specification to implement the part of the IS which enforces AC policy. This implementation step can be automated, by using some controller synthesis techniques or could be implemented by hands.

\section{Interpreting $\mathrm{EB}^{3} \mathrm{SEC}$ Models}

$\mathrm{EB}^{3} \mathrm{SEC}$ is based on the same process algebra as $\mathrm{EB}^{3}$ but extended with security environments. $\mathrm{EB}^{3}$ is implemented in a platform called APIS [18] that allows its interpretation. Components of this platform can be reused to create a Policy Decision Point (PDP), which enforces an AC policy. Figure 3 illustrates the architecture of the PDP.

$\mathrm{EB}^{3} \mathrm{PAI}$ [19] is an interpreter for $\mathrm{EB}^{3}$ process expressions. It can be used to run $\mathrm{EB}^{3} \mathrm{SEC}$ process expressions by translating secure events into $\mathrm{EB}^{3}$ events: 


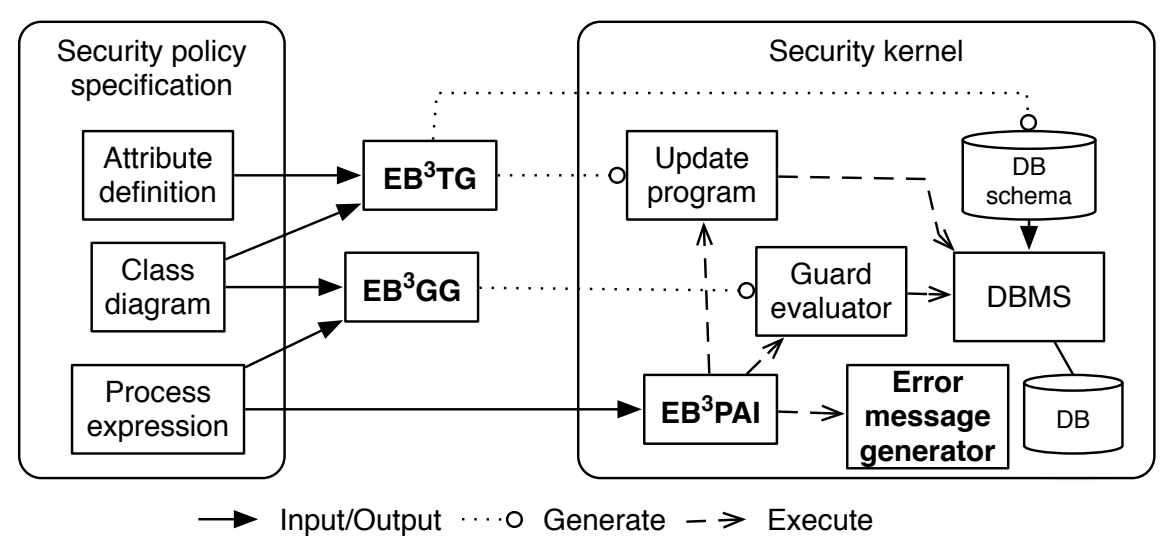

Fig. 3. Architecture of the PDP

$\left\langle p, r, o, t, \operatorname{evt}\left(a_{1}, \ldots, a_{n}\right)\right\rangle \triangleq s p(\langle p, r, o, t$, evt $\rangle) \Longrightarrow \operatorname{evt}^{\prime}\left(a_{1}, \ldots, a_{n}, p, r, o, t\right)$

Process expressions can contain predicates referring to attributes of the requirements class diagram and the security class diagram. The requirements class diagram deals with functional requirements: all classes and associations used by the IS without considering security issues. We suppose that each class diagram is implemented in its own relational database. To implement the security class diagram, $\mathrm{EB}^{3} \mathrm{TG}_{\text {generates a rela- }}$ tional database schema and update programs to implement attribute definitions. Update programs are used by $\mathrm{EB}^{3} \mathrm{PAI}$ to maintain the database in a consistent state while secure events are executed. The module $\mathrm{EB}^{3} \mathrm{GG}$ generates a guard evaluator program which contains procedures that are used by $\mathrm{EB}^{3} \mathrm{PAI}$ to determine if a guard holds. It generates SQL queries on the database to obtain attribute values of objects. These procedures can refer to both security and requirements class diagrams. If an event is rejected by $\mathrm{EB}^{3} \mathrm{PAI}$, the error message generator generates an adapted error message. SQL code to evaluate predicate $s p$ on a relational database implementing the security class diagram can also be generated .

$\mathrm{EB}^{3} \mathrm{PAI}$ can execute arbitrary process expressions and the state of process expressions is stored in a database. It can efficiently execute quantified expressions over arbitrary large sets (both choice and interleave) thanks to optimization techniques [19]. Its main weaknesses are that its OODB is rather slow and its execution cannot be easily distributed over several processors. For high throughput banking applications, this is not sufficient. Hence, we are currently working on a new process algebra interpreter which will be restricted to deterministic and optimizable process expressions. These process expressions can be translated into an algebraic state transition diagram (ASTD) [20]. The execution of an ASTD is easier to distribute over several processors, thereby exploiting parallelism in the processing of events. The state of an ASTD is more compact than the one in $\mathrm{EB}^{3} \mathrm{PAI}$ and can be easily stored into a relational database. 


\section{Related Work}

Many methods deal with modeling access control policies. A first point will be to find comparison criteria. The article [21] proposes a framework that provides a set of comparison points. $\mathrm{As} \mathrm{EB}^{3} \mathrm{SEC}$ is mainly an $\mathrm{AC}$ policy modeling language, only comparison points called model specification, policy expressiveness and policy language are relevant. The first point deals with the abstraction level of models (i.e., from implementation to requirements). The second point compares modeling methods by listing all concepts that can be expressed in the language. The last point compares languages on their syntax and formalisms.

$\mathrm{EB}^{3} \mathrm{SEC}$ allows $\mathrm{AC}$ policies to be modeled at an abstract level and deals with $\mathrm{AC}$ requirements. Many other languages, such as [10|9]22]12]23], also deals with requirements as input. Generally these languages have a fix metamodel. In other words, if the requirements do not comply with this metamodel, it could be tricky to achieve the modeling. OrBAC [10] has a new notion of organization but has also a fix metamodel. $\mathrm{EB}^{3} \mathrm{SEC}$ offers a flexible model to fit security requirements. Thereby, one can use a basic RBAC model in $\mathrm{EB}^{3} \mathrm{SEC}$ but extensions can be defined by modifying the security class diagram.

Once the metamodel is defined, concepts that can be used are also a criteria of comparison. Permission is a basic concept included in all AC policy modeling languages but only few of them propose the concept of prohibition [24|25|26]. Permissions and prohibitions can have constraints to restrict their application domain [27]12]. Static SoD is integrated in the RBAC model [9]15], thus all languages based on RBAC can also be used to express static SoD. Dynamic SoD is considered in [17]22]. In [28], mechanisms to define dynamic SoD in XACML are lightly described. The Soda language [29] is an AC policy modeling language that exclusively focuses on modeling SoD with algebra. The concept of obligation is defined in languages such as [4]. EB ${ }^{3}$ SEC can express permissions, prohibitions, both of them can have additional constraints, static and dynamic SoD and obligations.

Another relevant characteristic of AC policy modeling languages is their formal or semi-formal feature. Formal languages own a precise semantics and allow different kinds of analyses, such as theorem proving, model-checking or animation, to be carried out. This indubitably improves the quality of the resulting models. Methods based only on metamodels [30[10] are semi-formal by nature. The work of [31] aims at formalizing XACML, or a subset of, in order to achieve some verifications. Generally, existing formal methods adopt a state-based approach [17|32|33]. In other words, expressing dynamic AC constraints implies to define additional data necessary to memorize previous states of the system. Other formal methods [29|34|35] are based on process algebra, Petri nets or temporal logic but focuses on dynamic SoD constraints. $\mathrm{EB}^{3} \mathrm{SEC}$ is a formal language based on a process algebra that can express all kinds of $\mathrm{AC}$ constraints. $\mathrm{EB}^{3} \mathrm{SEC}$ specifications can be simulated by an interpreter (see section 4 ) and verification tools are under construction.

Most languages previously cited have an implementation. [16] describes how to transform a Soda model into a CSP specification to implement a monitor. XACML 
has two implementations 34 which are used in industrial contexts to enforce $\mathrm{AC}$ policies. MotOrBAC [36] is an implementation of OrBAC. We are currently working on an implementation of $\mathrm{EB}^{3} \mathrm{SEC}$ in a SOA architecture.

\section{Conclusion and Future Work}

This paper presents $\mathrm{EB}^{3} \mathrm{SEC}$, a formal language to express permissions, prohibitions, obligations and SoD constraints. Thanks to its high level of expressiveness, it allows the modeling of access control constraints at the workflow level in a unique framework. Another originality of $\mathrm{EB}^{3} \mathrm{SEC}$ is that the $\mathrm{AC}$ model is not fixed and can be adapted to the requirements of the IS. For instance, in the paper, we have chosen an OrBAC model extended with the concept of time. $\mathrm{EB}^{3} \mathrm{SEC}$ is currently tested in projects led with industrial partners from financial and healthcare domains. $\mathrm{EB}^{3} \mathrm{SEC}$ access control policies can be efficiently interpreted, by reusing components from the APIS platform supporting the $\mathrm{EB}^{3}$ method from which $\mathrm{EB}^{3} \mathrm{SEC}$ originates.

As a formal method, $\mathrm{EB}^{3} \mathrm{SEC}$ will allow one to use formal verification tools to verify a security policy. The next step in our work will be to develop a model-checker. As $\mathrm{EB}^{3} \mathrm{SEC}$ is derived from $\mathrm{EB}^{3}$, we plan to reuse results and tools developed for $\mathrm{EB}^{3}$. Alloy [37] will surely be the tool we will consider. The first step will be to transform $\mathrm{EB}^{3} \mathrm{SEC}$ models into Alloy models. To achieve this, the static part of an $\mathrm{EB}^{3} \mathrm{SEC}$ model (i.e.: the security class diagram) has to be transformed into an Alloy model. At this step we will be able to verify the consistency between permissions rules and prohibition rules. Then, we will need to transform the dynamic part (i.e.: obligations, SoD and more generally all PEs) in an Alloy model. Thus, we will be able to verify that dynamic and static parts are consistent.

Moreover, if a formal method is used to specify the functional part of an information system, especially $\mathrm{EB}^{3}$, it is also possible to verify the combination of the functional specification and the security rules. We also advocate a clear separation between the information system and its associated security policy, in order to have the ability to modify a security policy without having to modify the information system.

\section{References}

1. Société-Générale: Note explicative concernant la la fraude exceptionnelle (2008), http: //www . communiques-presse. net/Banque/societe-generale-noteexplicative-concernant-fraude-exceptionnel.html

2. Mer, F.: loi de sécurité financière. Journal Officiel (177) (January 2003)

3. Sarbanes, P., Oxley, M.: Sarbanes-oxley act. Public Law (116), 107-204 (2002)

4. Ni, Q., Bertino, E., Lobo, J.: An obligation model bridging access control policies and privacy policies. In: Proceedings of the 13th ACM Symposium on Access Control Models and Technologies. SACMAT 2008, pp. 133-142. ACM, New York (2008)

5. Ferraiolo, D.F., Kuhn, D.R., Chandramouli, R.: Role-Based Access Control. Artech House, Inc., Norwood (2003)

\footnotetext{
${ }^{3}$ http://code.google.com/p/enterprise-java-xacml/

${ }^{4} \mathrm{http} / / /$ sunxacml.sourceforge.net/
} 
6. Fraikin, B., Frappier, M., Laleau, R.: State-based versus event-based specifications for information systems: a comparison of B and $\mathrm{EB}^{3}$. Software and Systems Modeling 4(3), 236-257 (2005)

7. Frappier, M., St-Denis, R.: $\mathrm{EB}^{3}$ : an entity-based black-box specification method for information systems. Software and System Modeling 2(2), 134-149 (2003)

8. Bell, D.E., LaPadula, L.J.: Secure computer systems: Mathematical foundations and model. The MITRE Corporation Bedford MA Technical Report M74244 May 1(M74-244), 42 (1973)

9. International Committee for Information Technology Standards (INCITS) American National Standard for Information Technology (ANSI): Role-Based Access Control. 359-2004 edn (February 2004)

10. Kalam, A.A.E., Benferhat, S., Miège, A., Baida, R.E., Cuppens, F., Saurel, C., Balbiani, P., Deswarte, Y., Trouessin, G.: Organization based access control. In: Proceedings of the 4th IEEE International Workshop on Policies for Distributed Systems and Networks. POLICY 2003, IEEE Computer Society, Washington, DC, USA (2003)

11. Sandhu, R.S., Coyne, E.J., Feinstein, H.L., Youman, C.E.: Role-based access control models. IEEE Computer 29(2), 38-47 (1996)

12. Moses, T.: eXtensible Access Control Markup Langage (XACML) Version 2.0. OASIS Standard (2005)

13. Konopacki, P., Frappier, M., Laleau, R.: Expressing access control policies with an eventbased approach. Technical Report TR-LACL-2010-6, LACL (Laboratory of Algorithms, Complexity and Logic), University of Paris-Est, Paris 12 (2010), http://lacl.univ-paris12.fr/Rapports/TR/TR-LACL-2010-6.pdf

14. Anderson, A.: XACML Profile for Role Based Access Control (RBAC). OASIS Standard (2004)

15. Xin, J.: Applying model driven architecture approach to model role based access control system. Master's thesis, University of Ottawa (2006)

16. Basin, D., Burri, S.J., Karjoth, G.: Dynamic enforcement of abstract separation of duty constraints. In: Backes, M., Ning, P. (eds.) ESORICS 2009. LNCS, vol. 5789, pp. 250-267. Springer, Heidelberg (2009)

17. Miège, A.: Définition d'un environnement formel d'expression de politiques de sécurité. Modèle Or-BAC et extensions. PhD thesis, Paristech, ENST (September 2005)

18. Frappier, M., Fraikin, B., Gervais, F., Laleau, R., Richard, M.: Synthesizing information systems: the apis project. In: Rolland, C., Pastor, O., Cavarero, J.L. (eds.) RCIS, pp. 73-84 (2007)

19. Fraikin, B., Frappier, M.: Efficient symbolic computation of process expressions. Science of Computer Programming 74(9), 723-753 (2009)

20. Frappier, M., Gervais, F., Laleau, R., Fraikin, B., St-Denis, R.: Extending statecharts with process algebra operators. In: Innovations in Systems and Software Engineering, pp. 285292. Springer, London (August 2008)

21. Alm, C., Drouineaud, M., Faltin, U., Sohr, K., Wolf, R.: A classification framework designed for advanced role-based access control models and mechanisms. Technical report, Technologie-Zentrum Informatik Bremen University (2009)

22. Wainer, J., Barthelmess, P., Kumar, A.: W-rbac a workflow security model incorporating controlled overriding of constraints. International Journal of Cooperative Information Systems 12(4), 455-486 (2003)

23. Basin, D., Doser, J., Lodderstedt, T.: Model driven security: From uml models to access control infrastructures. ACM Trans. Softw. Eng. Methodol. 15(1), 39-91 (2006)

24. Bertino, E., Catania, B., Ferrari, E., Perlasca, P.: A logical framework for reasoning about access control models. In: Proceedings of the Sixth ACM Symposium on Access Control Models and Technologies. SACMAT 2001, ACM, New York (2001) 
25. Cholvy, L., Cuppens, F.: nalyzing consistency of security policies. In: Proceedings IEEE Symposium on Security and Privacy, pp. 103-112 (May 1997)

26. Jajodia, S., Samarati, P., Sapino, M.L., Subrahmanian, V.S.: Flexible support for multiple access control policies. ACM Trans. Database Syst. 26, 214-260 (2001)

27. Bertino, E., Bonatti, P.A., Ferrari, E.: Trbac: A temporal role-based access control model. ACM Trans. Inf. Syst. Secur. 4, 191-233 (2001)

28. Crampton, J., Khambhammettu, H.: Xacml and role-based access control. In: Presentation at DIMACS Workshop on Security of Web Services and e-Commerce, p. 174. Springer, Heidelberg (2005)

29. Li, N., Wang, Q.: Beyond separation of duty: An algebra for specifying high-level security policies. J. ACM 55(3), 1-46 (2008)

30. Lodderstedt, T., Basin, D.A., Doser, J.: Secureuml: A uml-based modeling language for model-driven security. In: Proceedings of the 5th International Conference on The Unified Modeling Language. UML 002, London, UK, pp. 426-441. Springer, Heidelberg (2002)

31. Kolovski, V., Hendler, J., Parsia, B.: Analyzing web access control policies. In: WWW 2007: Proceedings of the 16th International Conference on World Wide Web, pp. 677-686. ACM, New York (2007)

32. Sohr, K., Drouineaud, M., Ahn, G.J., Gogolla, M.: Analyzing and managing role-based access control policies. IEEE Trans. on Knowl. and Data Eng. 20, 924-939 (2008)

33. Basin, D., Clavel, M., Doser, J., Egea, M.: Automated analysis of security-design models. Inf. Softw. Technol. 51, 815-831 (2009)

34. Ayed, S., Cuppens-Boulahia, N., Cuppens, F.: Deploying access control in distributed workflow. In: Proceedings of the Sixth Australasian Conference on Information Security, Darlinghurst, Australia. AISC 2008, vol. 81, pp. 9-17. Australian Computer Society, Inc. (2008)

35. Kallel, S., Charfi, A., Mezini, M., Jmaiel, M., Klose, K.: From formal access control policies to runtime enforcement aspects. In: Massacci, F., Redwine Jr., S.T., Zannone, N. (eds.) ESSoS 2009. LNCS, vol. 5429, pp. 16-31. Springer, Heidelberg (2009)

36. Cuppens, F., Cuppens-Boulahia, N., Coma, C.: MotOrBAC: un outil d'administration et de simulation de politiques de sécurité. In: Security in Network Architectures (SAR) and Security of Information Systems (SSI), First Joint Conference, June 6-9 (2006)

37. Jackson, D.: Software Abstractions: Logic, Language, and Analysis. The MIT Press, Cambridge (2006) 